\title{
Exact Null Controllability for Fractional Nonlocal Integrodifferential Equations via Implicit Evolution System
}

\author{
Amar Debbouche $^{1}$ and Dumitru Baleanu' ${ }^{2,3}$ \\ ${ }^{1}$ Department of Mathematics, Faculty of Science, Guelma University, Guelma, Algeria \\ ${ }^{2}$ Department of Mathematics and Computer Science, Cankaya University, Ankara, Turkey \\ ${ }^{3}$ Institute of Space Sciences, P.O. Box, MG-23, 76900 Magurele-Bucharest, Romania
}

Correspondence should be addressed to Dumitru Baleanu, dumitru@cankaya.edu.tr

Received 1 May 2012; Revised 15 July 2012; Accepted 16 July 2012

Academic Editor: F. Marcellán

Copyright (C) 2012 A. Debbouche and D. Baleanu. This is an open access article distributed under the Creative Commons Attribution License, which permits unrestricted use, distribution, and reproduction in any medium, provided the original work is properly cited.

We introduce a new concept called implicit evolution system to establish the existence results of mild and strong solutions of a class of fractional nonlocal nonlinear integrodifferential system, then we prove the exact null controllability result of a class of fractional evolution nonlocal integrodifferential control system in Banach space. As an application that illustrates the abstract results, two examples are provided.

\section{Introduction}

In this paper, we study the fractional nonlocal integrodifferential system of the form

$$
\begin{gathered}
\frac{d^{\alpha} u(t)}{d t^{\alpha}}+A\left(t, B_{1} u(t)\right) u(t)=f\left(t, B_{2} u(t)\right)+\int_{0}^{t} g\left(t, s, B_{3} u(s)\right) d s, \\
B_{4}\left(u(0)-u_{0}\right)=\int_{0}^{a} h(u(t)) d t,
\end{gathered}
$$

where $0<\alpha \leq 1, t \in[0, a]$. Let $-A$ be the infinitesimal generator of a $C_{0}$-semigroup in a Banach space $X$, and $\left\{B_{i}(t): i=1,2,3,4\right\}$ is a family of linear closed operators defined on dense sets $S_{i} \supset D(A), i=1,2,3,4$, respectively, in $X$ into $X$. It is assumed that $u_{0} \in X$, 
$f: I \times X \rightarrow X, g: \Delta \times X \rightarrow X$ and $h: C(I: X) \rightarrow X$ are given abstract functions. Here, $I=[0, a]$ and $\Delta=\{(s, t): 0 \leq s \leq t \leq a\}$.

Basic researches in differential equations have showed that many phenomena in nature are modeled more accurately using fractional derivatives and integrals; for more detail, we can refer to [1-13] and the references therein. There are many applications where the fractional calculus can be used, for example, viscoelasticity, electrochemistry, diffusion processes, control theory, heat conduction, electricity, mechanics, chaos, and fractals [14].

Controllability is a fundamental concept in mathematical control theory and plays an important role in both finite and infinite dimensional spaces, that is, systems represented by ordinary differential equations and partial differential equations, respectively. So it is natural to extend this concept to dynamical systems represented by fractional differential equations. Several fractional partial differential equations and integrodifferential equations can be expressed abstractly in some Banach spaces, in many cases, the accurate analysis, design and assessment of systems subjected to realistic environments must take into account the potential of random loads and randomness in the system properties. Randomness is intrinsic to the mathematical formulation of many phenomena such as fluctuations in the stock market or noise in communication networks. Fu studied the controllability results of some kinds of neutral functional differential systems, see [15, 16]. In our previous work [17], we established the controllability of fractional evolution nonlocal impulsive quasilinear delay integrodifferential systems.

The existence results to evolution equations with nonlocal conditions in Banach space were studied first by Byszewski $[18,19]$; subsequently, many authors have been studied the same question, see for instance [20-23].

Deng [24] indicated that, using the nonlocal condition $u(0)+h(u)=u_{0}$ to describe for instance, the diffusion phenomenon of a small amount of gas in a transparent tube can give better result than using the usual local Cauchy problem $u(0)=u_{0}$. Let us observe also that, since Deng's papers, the function $h$ is considered

$$
h(u)=\sum_{k=1}^{p} c_{k} u\left(t_{k}\right)
$$

where $c_{k}, k=1,2, \ldots, p$ are given constants and $0 \leq t_{1}<\cdots<t_{p} \leq a$.

In this paper, we introduce a new concept in the theory of Semigroup named "implicit evolution system" to show the reader "what is the main difference between the solutions of fractional $(0<\alpha<1)$ and classical (first order) homogeneous evolution equation?" which is based on the work [17] and Pazy [25]. A new form of nonlocal condition is also presented.

Our paper is organized as follows. Section 2 is devoted to a review of some essential results which will be used in this work to obtain our main results. In Section 3, we use the theory of semigroups [25] in order to introduce our new concept that is called implicit evolution system. In Section 4, we establish the existence, uniqueness, and regularity of mild solutions of a class of fractional evolution nonlinear integrodifferential systems with nonlocal conditions in Banach space. In Section 5, we prove the exact null controllability of a class of fractional evolution nonlocal integrodifferential control systems; the last section deals to give examples that provide the abstract results. 


\section{Preliminary Results}

Definition 2.1. The fractional integral of order $\alpha$ with the lower limit zero for a function $f \in$ $C([0, \infty))$ is defined as

$$
I^{\alpha} f(t)=\frac{1}{\Gamma(\alpha)} \int_{0}^{t} \frac{f(s)}{(t-s)^{1-\alpha}} d s, \quad t>0,0<\alpha<1
$$

provided the right side is pointwise defined on $[0, \infty)$, where $\Gamma$ is the gamma function.

Riemann-Liouville derivative of order $\alpha$ with the lower limit zero for a function $f \in$ $C([0, \infty))$ can be written as

$$
{ }^{L} D^{\alpha} f(t)=\frac{1}{\Gamma(1-\alpha)} \frac{d}{d t} \int_{0}^{t} \frac{f(s)}{(t-s)^{\alpha}} d s, \quad t>0,0<\alpha<1 .
$$

The Caputo derivative of order $\alpha$ for a function $f \in C([0, \infty))$ can be written as

$$
{ }^{C} D^{\alpha} f(t)={ }^{L} D^{\alpha}(f(t)-f(0)), \quad t>0,0<\alpha<1 .
$$

Remark 2.2. (1) If $f \in C^{1}([0, \infty))$, then

$$
{ }^{C} D^{\alpha} f(t)=\frac{1}{\Gamma(1-\alpha)} \int_{0}^{t} \frac{f^{\prime}(s)}{(t-s)^{\alpha}} d s=I^{1-\alpha} f^{\prime}(t), \quad t>0,0<\alpha<1 .
$$

(2) The Caputo derivative of a constant is equal to zero.

(3) If $f$ is an abstract function with values in $X$, then integrals which appear in Definition 2.1 are taken in Bochner's sense.

Definition 2.3. By a strong solution of the nonlocal Cauchy problem (1.1), (1.2), we mean a function $u$ with values in $X$ such that

(i) $u$ is a continuous function in $t \in I$ and $u(t) \in D(A)$,

(ii) $d^{\alpha} u / d t^{\alpha}$ exists and continuous on $(0, a], 0<\alpha<1$, and $u$ satisfies $(1.1)$ on $(0, a]$ and (1.2).

It is suitable to rewrite (1.1), (1.2) in the form

$$
\begin{aligned}
u(t)= & u(0)+\frac{1}{\Gamma(\alpha)} \int_{0}^{t}(t-\eta)^{\alpha-1} \\
& \times\left[-A\left(\eta, B_{1} u(\eta)\right) u(\eta)+f\left(\eta, B_{2} u(\eta)\right)+\int_{0}^{\eta} g\left(\eta, s, B_{3} u(s)\right) d s\right] d \eta,
\end{aligned}
$$

see also $[26,27]$.

Let $X$ and $Y$ be two Banach spaces such that $Y$ is densely and continuously embedded in $X$. We denote by $Z$ every Banach space $Z(I, X)$ endowed with the usual norm, which is given by $\|u\|_{Z}=\sup _{t \in I}\|u(t)\|$, for $u \in Z$. The space of all bounded linear operators from $X$ to $Y$ is denoted by $B(X, Y)$. We recall some definitions and known facts from Pazy [25]. 
Definition 2.4. Let $S$ be a linear operator in $X$, and let $Y$ be a subspace of $X$. The operator $\widetilde{S}$ defined by $D(\widetilde{S})=\{x \in D(S) \cap Y: S x \in Y\}$ and $\widetilde{S} x=S x$ for $x \in D(\widetilde{S})$ is called the part of $S$ in $Y$.

Definition 2.5. Let $\Omega$ be a subset of $X$ and for every $t \in I$ and $B_{1} u \in \Omega$, and let $-A\left(t, B_{1} u\right)$ be the infinitesimal generator of a $C_{0}$-semigroup $S_{t, B_{1} u}(s), s \geq 0$, on $X$. The family of operators $\left\{A\left(t, B_{1} u\right),\left(t, B_{1} u\right) \in I \times \Omega\right\}$ is stable if there are constants $M \geq 1$ and $\omega$ such that

$$
\begin{gathered}
\rho\left(A\left(t, B_{1} u\right)\right) \supset(\omega, \infty) \\
\left\|\prod_{j=1}^{k} R\left(\lambda: A\left(t_{j}, B_{1} u\right)\right)\right\| \leq M(\lambda-\omega)^{-k}
\end{gathered}
$$

for $\lambda>\omega$ every finite sequences $0 \leq t_{1} \leq t_{2} \leq \cdots \leq t_{k} \leq a, 1 \leq j \leq k$.

The stability of $\left\{A\left(t, B_{1} u\right),\left(t, B_{1} u\right) \in I \times \Omega\right\}$ implies that

$$
\left\|\prod_{j=1}^{k} S_{t_{j}, B_{1} u}\left(s_{j}\right)\right\| \leq M \exp \left(\omega \sum_{j=1}^{k} s_{j}\right), \quad s_{j} \geq 0,
$$

and any finite sequences $0 \leq t_{1} \leq t_{2} \leq \cdots \leq t_{k} \leq a, 1 \leq j \leq k, k=1,2, \ldots$

Definition 2.6. Let $S_{t, B_{1} u}(s), s \geq 0$ be the $C_{0}$-semigroup generated by $A\left(t, B_{1} u\right),\left(t, B_{1} u\right) \in I \times \Omega$. A subspace $Y$ of $X$ is called $A\left(t, B_{1} u\right)$-admissible if $Y$ is invariant subspace of $S_{t, B_{1} u}(s)$, and the restriction of $S_{t, B_{1} u}(s)$ to $Y$ is a $C_{0}$-semigroup in $Y$.

Let $\Omega \subset X$ be a subset of $X$ such that for every $\left(t, B_{1} u\right) \in I \times \Omega, A\left(t, B_{1} u\right)$ is the infinitesimal generator of a $C_{0}$-semigroup $S_{t, B_{1} u}(s), s \geq 0$ on $X$. We make the following assumptions.

$\left(\mathrm{H}_{1}\right)$ The family $\left\{A\left(t, B_{1} u\right),\left(t, B_{1} u\right) \in I \times \Omega\right\}$ is stable.

$\left(\mathrm{H}_{2}\right) Y$ is $A\left(t, B_{1} u\right)$-admissible for $\left(t, B_{1} u\right) \in I \times \Omega$, and the family $\left\{\tilde{A}\left(t, B_{1} u\right),\left(t, B_{1} u\right) \in\right.$ $I \times \Omega\}$ of parts $\widetilde{A}\left(t, B_{1} u\right)$ of $A\left(t, B_{1} u\right)$ in $Y$ is stable in $Y$.

$\left(\mathrm{H}_{3}\right)$ For $\left(t, B_{1} u\right) \in I \times \Omega, D\left(A\left(t, B_{1} u\right)\right) \supset Y, A\left(t, B_{1} u\right)$ is a bounded linear operator from $Y$ to $X$ and $t \rightarrow A\left(t, B_{1} u\right)$ is continuous in the $B(Y, X)$ norm $\|\cdot\|$.

$\left(\mathrm{H}_{4}\right)$ There is a constant $L>0$ such that

$$
\left\|A\left(t, B_{1} u\right)-A\left(t, B_{1} v\right)\right\|_{Y \rightarrow X} \leq L\|u-v\|_{X}
$$

holds for every $B_{1} u, B_{1} v \in \Omega$, and $t \in I$.

In the next section, we will introduce a new concept in the theory of semigroups.

\section{Implicit Evolution System}

Let $\Omega$ be a subset of $X$ and $\left\{A\left(t, B_{1} u\right),\left(t, B_{1} u\right) \in I \times \Omega\right\}$ a family of operators satisfying the conditions $\left(\mathrm{H}_{1}\right)-\left(\mathrm{H}_{4}\right)$. If $u \in C(I: X)$ has values in $\Omega$, then there is a unique evolution system $U_{\alpha}\left(t, s ; B_{1} u\right), 0<\alpha \leq 1,0 \leq s \leq t \leq a$, in $X$ satisfying 
(i) $\left\|U_{\alpha}\left(t, s ; B_{1} u\right)\right\| \leq M e^{\omega(t-s)}$ for $0 \leq s \leq t \leq a$, where $M$ and $\omega$ are stability constants,

(ii) $\left(\partial^{\alpha} / \partial t^{\alpha}\right) U_{\alpha}\left(t, s ; B_{1} u\right) y=A\left(s, B_{1} u(s)\right) U_{\alpha}\left(t, s ; B_{1} u\right) y$ for $y \in Y$ and $0 \leq s \leq t \leq a$,

(iii) $\left(\partial^{\alpha} / \partial s^{\alpha}\right) U_{\alpha}\left(t, s ; B_{1} u\right) y=-U_{\alpha}\left(t, s ; B_{1} u\right) A\left(s, B_{1} u(s)\right) y$ for $y \in Y$ and $0 \leq s \leq t \leq a$.

Remark 3.1. (1) If $B_{1}$ is the identity and $\alpha=1$, then $U(t, s ; u)$ is the explicit evolution system given in Pazy [25] and in Zaidman [28].

(2) Since, in our case, $U(t, s ; u)$ is dependent of $\alpha$ and $B_{1}$, so we call it an implicit evolution system generated by $-A\left(t, B_{1} u\right)$.

(3) For nonautonomous differential equations in a Banach space, the implicit evolution system is similar to our concept $(\alpha, u)$-resolvent family.

(4) We can deduce that (1.1)-(1.2) is well posed if and only if $-A\left(t, B_{1} u\right)$ is the generator of the implicit evolution system $U(t, s ; u)$.

Further, we assume the following.

$\left(\mathrm{H}_{5}\right)$ For every $u \in C(I: X)$ satisfying $u(t) \in \Omega$ for $0 \leq t \leq a$, we have

$$
U(t, s ; u) Y \subset Y, \quad 0 \leq s \leq t \leq a
$$

and $U(t, s ; u)$ is strongly continuous in $Y$ for $0 \leq s \leq t \leq a$.

$\left(\mathrm{H}_{6}\right) Y$ is reflexive.

$\left(\mathrm{H}_{7}\right)$ For every $\left(t, B_{2} u\right) \in I \times \Omega, f\left(t, B_{2} u\right) \in Y$.

$\left(\mathrm{H}_{8}\right)$ The operator $\left[B_{4}(t)+\lambda^{\alpha} I\right]^{-1}$ exists in $B(X)$ for any $\mathcal{l}$ with $\operatorname{Re} \lambda \leq 0$ and

$$
\left\|\left[B_{4}(t)+\lambda^{\alpha} I\right]^{-1}\right\| \leq \frac{C_{\alpha}}{|\lambda|+1}, \quad t \in I,
$$

where $C_{\alpha}$ is a positive constant independent of both $t$ and $\lambda$.

$\left(\mathrm{H}_{9}\right) h: C(I: \Omega) \rightarrow Y$ is Lipschitz continuous in $X$ and bounded in $Y$, that is, there exist constants $k_{1}>0$ and $k_{2}>0$ such that

$$
\begin{gathered}
\|h(u)\|_{Y} \leq k_{1} \\
\|h(u)-h(v)\|_{Y} \leq k_{2} \max _{t \in I}\|u-v\|_{X} .
\end{gathered}
$$

For the conditions $\left(\mathrm{H}_{9}\right)$ and $\left(\mathrm{H}_{10}\right)$, let $Z$ be taken as both $X$ and $Y$.

$\left(\mathrm{H}_{10}\right) g: \Delta \times Z \rightarrow Z$ is continuous, and there exist constants $k_{3}>0$ and $k_{4}>0$ such that

$$
\begin{gathered}
\int_{0}^{t}\left\|g\left(t, s, B_{3} u\right)-g\left(t, s, B_{3} v\right)\right\|_{Z} d s \leq k_{3}\|u-v\|_{Z}, \quad u, v \in X, \\
k_{4}=\max \left\{\int_{0}^{t}\|g(t, s, 0)\|_{Z} d s:(t, s) \in \Delta\right\} .
\end{gathered}
$$



that

$\left(\mathrm{H}_{11}\right) f: I \times Z \rightarrow Z$ is continuous, and there exist constants $k_{5}>0$ and $k_{6}>0$ such

$$
\begin{gathered}
\left\|f\left(t, B_{2} u\right)-f\left(t, B_{2} v\right)\right\|_{Z} \leq k_{5}\|u-v\|_{Z}, \quad u, v \in X, \\
k_{6}=\max _{t \in I}\|f(t, 0)\|_{Z} .
\end{gathered}
$$

Let us take $M_{0}=\max \|U(t, s ; u)\|_{\Omega(Z)}, 0 \leq s \leq t \leq a, u \in \Omega$.

$\left(\mathrm{H}_{12}\right)$ There exist positive constants $r>0$ and $0<\lambda<1$ such that

$$
\begin{gathered}
M_{0}\left\{\left\|u_{0}\right\|+a C_{\alpha} k_{1}+a\left[r\left(k_{3}+k_{5}\right)+k_{4}+k_{6}\right]\right\} \leq r \\
\mathcal{\lambda}=K a\left\|u_{0}\right\|_{Y}+a^{2} C_{\alpha} k_{1} K+a M_{0} C_{\alpha} k_{2}+a\left\{K\left[\left(k_{3}+k_{5}\right) r+k_{4}+k_{6}\right] a+M_{0}\left(k_{3}+k_{5}\right)\right\} .
\end{gathered}
$$

By a mild solution of (1.1), (1.2), we mean a function $u \in C(I: X)$ with values in $\Omega$ and $u_{0} \in X$ satisfying the integral equation

$$
\begin{aligned}
u(t)= & U(t, 0 ; u) u_{0}+U(t, 0 ; u) B_{4}^{-1} \int_{0}^{a} h(u(t)) d t \\
& +\int_{0}^{t} U(t, s ; u)\left[f\left(s, B_{2} u(s)\right)+\int_{0}^{s} g\left(s, \eta, B_{3} u(\eta)\right) d \eta\right] d s .
\end{aligned}
$$

$\left(\mathrm{H}_{13}\right)$ Further, there exists a constant $K>0$ such that for every $u, v \in C(I: X)$ with values in $\Omega$ and every $\omega \in Y$ we have

$$
\|U(t, s ; u) \omega-U(t, s ; v) \omega\| \leq K\|\omega\|_{Y} \int_{s}^{t}\|u(\tau)-v(\tau)\| d \tau
$$

\section{Existence Results}

Theorem 4.1. Let $u_{0} \in Y$ and $\Omega=\left\{u \in X:\|u\|_{Y} \leq r\right\}, r>0$. If $-A\left(t, B_{1} u\right)$ is the generator of an implicit evolution system $U(t, s ; u)$ and the assumptions $\left(H_{5}\right) \sim\left(H_{13}\right)$ are satisfied, then (1.1), (1.2) has a unique mild solution on $I$.

Proof. Let $S$ be a nonempty closed subset of $C(I: X)$ defined by

$$
S=\left\{u: u \in C(I: X),\|u\|_{Y} \leq r\right\}, \quad t \in I
$$


Consider a mapping $P$ on $S$ defined by

$$
\begin{aligned}
(P u)(t)= & U(t, 0 ; u) u_{0}+U(t, 0 ; u) B_{4}^{-1} \int_{0}^{a} h(u(t)) d t \\
& +\int_{0}^{t} U(t, s ; u)\left[f\left(s, B_{2} u(s)\right)+\int_{0}^{s} g\left(s, \eta, B_{3} u(\eta)\right) d \eta\right] d s .
\end{aligned}
$$

For $u \in S$, we have

$$
\begin{aligned}
\|P u(t)\|_{Y} \leq & \left\|U(t, 0 ; u) u_{0}\right\|+\left\|U(t, 0 ; u) B_{4}^{-1} \int_{0}^{a} h(u(t)) d t\right\| \\
& +\int_{0}^{t}\|U(t, s ; u)\|\left\{\left\|f\left(s, B_{2} u(s)\right)-f(s, 0)\right\|+\|f(s, 0)\|\right. \\
& \left.+\left\|\int_{0}^{s}\left[g\left(s, \eta, B_{3} u(\eta)\right)-g(s, \eta, 0)\right] d \eta\right\|+\left\|\int_{0}^{s} g(s, \eta, 0) d \eta\right\|\right\} d s . \\
\leq & M_{0}\left\|u_{0}\right\|+a M_{0} C_{\alpha} k_{1} \\
& +\int_{0}^{t} M_{0}\left\{k_{5}\|u(s)\|+k_{6}+k_{3}\|u(s)\|+k_{4}\right\} d s \\
\leq & M_{0}\left\|u_{0}\right\|+a M_{0} C_{\alpha} k_{1}+a M_{0}\left\{k_{5}\|u(s)\|+k_{6}+k_{3}\|u(s)\|+k_{4}\right\} \\
\leq & M_{0}\left\{\left\|u_{0}\right\|+a C_{\alpha} k_{1}+a\left[r\left(k_{3}+k_{5}\right)+k_{4}+k_{6}\right]\right\} \\
\leq & r .
\end{aligned}
$$

Thus, $P$ maps $S$ into itself. Now, we will show that $P$ is a strict contraction on $S$ which will ensure the existence of a unique continuous function satisfying (3.7) on $I$.

If $u, v \in S$, then

$$
\begin{aligned}
& \|P u(t)-P v(t)\| \\
& \leq\left\|U(t, 0 ; u) u_{0}-U(t, 0 ; v) u_{0}\right\|+\left\|U(t, 0 ; u) B_{4}^{-1} \int_{0}^{a} h(u(t)) d t-U(t, 0 ; v) B_{4}^{-1} \int_{0}^{a} h(v(t)) d t\right\| \\
& \quad+\int_{0}^{t} \| U(t, s ; u)\left[f\left(s, B_{2} u(s)\right)+\int_{0}^{s} g\left(s, \eta, B_{3} u(\eta)\right) d \eta\right] \\
& \quad-U(t, s ; v)\left[f\left(s, B_{2} v(s)\right)+\int_{0}^{s} g\left(s, \eta, B_{3} v(\eta)\right) d \eta\right] \| d s \\
& \leq\left\|U(t, 0 ; u) u_{0}-U(t, 0 ; v) u_{0}\right\|+\left\|U(t, 0 ; u) B_{4}^{-1} \int_{0}^{a} h(u(t)) d t-U(t, 0 ; v) B_{4}^{-1} \int_{0}^{a} h(u(t)) d t\right\| \\
& \quad+\left\|U(t, 0 ; v) B_{4}^{-1} \int_{0}^{a} h(u(t)) d t-U(t, 0 ; v) B_{4}^{-1} \int_{0}^{a} h(v(t)) d t\right\|
\end{aligned}
$$




$$
\begin{gathered}
+\int_{0}^{t}\left\{\| U(t, s ; u)\left[f\left(s, B_{2} u(s)\right)+\int_{0}^{s} g\left(s, \eta, B_{3} u(\eta)\right) d \eta\right]\right. \\
-U(t, s ; v)\left[f\left(s, B_{2} u(s)\right)+\int_{0}^{s} g\left(s, \eta, B_{3} u(\eta)\right) d \eta\right] \| \\
+\| U(t, s ; v)\left[f\left(s, B_{2} u(s)\right)+\int_{0}^{s} g\left(s, \eta, B_{3} u(\eta)\right) d \eta\right] \\
\left.-U(t, s ; v)\left[f\left(s, B_{2} v(s)\right)+\int_{0}^{s} g\left(s, \eta, B_{3} v(\eta)\right) d \eta\right] \|\right\} d s \\
+K a u_{0}\left\|_{Y} \max _{\tau \in I}\right\| u(\tau)-v(\tau)\left\|+a^{2} C_{\alpha} k_{1} K \max _{\tau \in I}\right\| u(\tau)-v(\tau) \| \\
+M_{0} C_{\alpha} k_{2} \max _{\tau \in I}^{t}\{K\|u(\tau)-v(\tau)\| \\
+\int_{0}\left(s, B_{2} u(s)\right)+\int_{0}^{s} g\left(s, \eta, B_{3} u(\eta)\right) d \eta\left\|_{Y} a \max _{\tau \in I}\right\| u(\tau)-v(\tau) \| \\
+M_{0}\left[\left\|f\left(s, B_{2} u(s)\right)-f\left(s, B_{2} v(s)\right)\right\|\right. \\
\left.\left.+\int_{0}^{s}\left\|g\left(s, \eta, B_{3} u(\eta)\right)-g\left(s, \eta, B_{3} v(\eta)\right)\right\| d \eta\right]\right\} d s \\
\leq\left(K a\left\|u_{0}\right\|_{Y}+a^{2} C_{\alpha} k_{1} K+a M_{0} C_{\alpha} k_{2}\right) \max _{\tau \in I}\|u(\tau)-v(\tau)\| \\
+a\left\{K\left[\left(k_{3}+k_{5}\right) r+k_{4}+k_{6}\right] a+M_{0}\left(k_{3}+k_{5}\right)\right\} \max _{\tau \in I}\|u(\tau)-v(\tau)\| .
\end{gathered}
$$

Thus,

$$
\|P u(t)-P v(t)\| \leq \lambda \max _{\tau \in I}\|u(\tau)-v(\tau)\|
$$

which means that $P$ is a strict contraction map from $S$ into $S$, and therefore by the Banach contraction principle there exists a unique fixed point $u \in S$ such that $P u=u$. Hence, $u$ is a unique mild solution of (1.1), (1.2) on $I$.

Theorem 4.2. Assume the following.

(i) Conditions $\left(H_{1}\right) \sim\left(H_{13}\right)$ hold.

(ii) The functions $B_{2}(t) \omega$ and $B_{3}(t) \omega$ are uniformly Hölder continuous in $t \in I$ for every element $\omega$ in $S_{2} \cap S_{3}$.

(iii) There are numbers $L_{1}, L_{2}>0$ and $p, q \in(0,1]$ such that 
Journal of Applied Mathematics

$$
\begin{gathered}
\qquad \begin{array}{l}
\left\|f\left(t_{1}, B_{2} u\right)-f\left(t_{2}, B_{2} v\right)\right\| \leq L_{1}\left(\left|t_{1}-t_{2}\right|^{p}+\left\|B_{2} u-B_{2} v\right\|\right), \\
\left\|g\left(s_{1}, \eta, B_{3} u\right)-g\left(s_{2}, \eta, B_{3} v\right)\right\| \leq L_{2}\left|s_{1}-s_{2}\right|^{q}
\end{array} \\
\text { for all } t_{1}, t_{2} \in I \text { and all }\left(s_{1}, \eta\right),\left(s_{2}, \eta\right) \in \Delta .
\end{gathered}
$$

Then, the problem (1.1), (1.2) has a unique strong solution on I.

Proof. Applying Theorem 4.1, the problem (1.1), (1.2) has a mild solution $u \in S$. Now, we will show that $u$ is a unique strong solution of the considered problem on $I$.

According to (ii), $\left\|B_{2} u-B_{2} v\right\|$ is uniformly Hölder continuous in $t \in I$ for every element $u$ in $S_{2} \cap S_{3}$, also (iii) implies that $t \rightarrow f\left(t, B_{2} u\right)$ and $t \rightarrow \int_{0}^{t} g\left(t, s, B_{3} u\right) d s$ are uniformly Hölder continuous on $I([20,26])$.

Set

$$
V(t)=f\left(t, B_{2} u\right)+\int_{0}^{t} g\left(t, s, B_{3} u\right) d s
$$

Clearly $V(t)$ is uniformly Hölder continuous in $t \in I$.

Consider the following nonlocal Cauchy problem:

$$
\begin{gathered}
\frac{d^{\alpha} v(t)}{d t^{\alpha}}+A\left(t, B_{1} u(t)\right) u(t)=V(t), \\
B_{4}\left(u(0)-u_{0}\right)=\int_{0}^{a} h(u(t)) d t .
\end{gathered}
$$

From Pazy, (4.8) has a unique solution $v$ on $I$ given by

$$
v(t)=U(t, 0 ; u) u_{0}+U(t, 0 ; u) B_{4}^{-1} \int_{0}^{a} h(u(t)) d t+\int_{0}^{t} U(t, s ; u) V(s) d s
$$

Noting that [21], each term on the right hand side of (4.9) belongs to $D(A)$, thus $v(t) \in D(A)$, using the uniqueness of $V(t)$, we have that $u(t)=v(t)$. Hence, $u$ is the unique strong solution of (1.1), (1.2) on $I$.

In next section, some results are obtained from Sakthivel et al. [29, 30].

\section{Exactly Null Controllability Results}

Consider fractional nonlocal evolution integrodifferential control system of the form

$$
\begin{gathered}
\frac{d^{\alpha} u(t)}{d t^{\alpha}}+A\left(t, B_{1} u(t)\right) u(t)=(\Phi \mu)(t)+\Psi\left(t, f\left(t, B_{2} u(t)\right), \int_{0}^{t} g\left(t, s, B_{3} u(s)\right) d s\right) \\
B_{4}\left(u(0)-u_{0}\right)=\int_{0}^{a} h(u(t)) d t
\end{gathered}
$$


where the unknown $u(\cdot)$ takes values in the Banach space $X$, the control function $\mu$ belongs to the spaces $L^{2}(I, H)$, a Banach space of admissible control functions with $H$, a Banach space. Further, $\Phi$ is a bounded linear operator from $H$ into $X$, the function $\Psi: I \times X \times X \rightarrow X$ is given, and the others terms are defined as above.

For all $u_{0} \in X$ and admissible control $\mu \in L^{2}(I, H)$, the problem (5.1) admits a mild solution given by

$$
\begin{aligned}
u_{\mu}(t)= & U(t, 0 ; u) u_{0}+U(t, 0 ; u) B_{4}^{-1} \int_{0}^{a} h(u(t)) d t \\
& +\int_{0}^{t} U(t, s ; u)\left[\Phi \mu(s)+\Psi\left(s, f\left(s, B_{2} u(s)\right), \int_{0}^{s} g\left(s, \tau, B_{3} u(\tau)\right) d \tau\right)\right] d s .
\end{aligned}
$$

Definition 5.1. We will say that system (5.1) is exactly null controllable on the interval $I$ if for a11 $u_{0} \in X$, there exists a control $\mu \in L^{2}(I, H)$, such that the mild solution $u(t)$ of (5.1) corresponding to $\mu$ verifies $u(0)-B_{4}^{-1} \int_{0}^{a} h(u(t)) d t=u_{0}$ and $u(a)=0$.

In order to prove the controllability result, in addition, we consider the following conditions.

$\left(\mathrm{H}_{14}\right) \Psi: I \times X \times X \rightarrow X$ is continuous, and there exist constants $N_{1}$ and $N_{2}$ such that for all $x_{i}, y_{i} \in X, i=1,2$, we have

$$
\begin{gathered}
\left\|\Psi\left(t, x_{1}, y_{1}\right)-\Psi\left(t, x_{2}, y_{2}\right)\right\| \leq N_{1}\left[\left\|x_{1}-x_{2}\right\|+\left\|y_{1}-y_{2}\right\|\right] \\
N_{2}=\max _{t \in I}\|\Psi(t, 0,0)\| .
\end{gathered}
$$

$\left(\mathrm{H}_{15}\right)$ Let

$$
\rho+a M_{0} M_{1} M_{2}(\rho+\sigma)+\sigma \leq r
$$

where $\sigma=a M_{0}\left\{N_{1}\left[\left(k_{3}+k_{5}\right) r+k_{4}+k_{6}\right]+N_{2}\right\}$ and $\rho=M_{0}\left\|u_{0}\right\|+a M_{0} C_{\alpha} k_{1}$, and let

$$
\lambda=\left\{K a\left\|u_{0}\right\|+a^{2} C_{\alpha} k_{1} K+a M_{0} C_{\alpha} k_{2}\right\}+2 a\left\{M_{0} M_{1} M_{2}(\rho+a p)\right\}+a\{K q a+p\},
$$

where $p=M_{0} N_{1}\left(k_{3}+k_{5}\right), q=N_{1}\left[\left(k_{3}+k_{5}\right) r+k_{4}+k_{6}\right]+N_{2}$, and $0 \leq \lambda<1$.

$\left(\mathrm{H}_{16}\right)$ The bounded linear operator $W: L^{2}(I, H) \rightarrow X$ defined by

$$
W \mu=\int_{0}^{a} U(a, s ; u) \Phi \mu d s
$$

has an induced inverse operator $\widetilde{W}^{-1}$ which takes values in $L^{2}(I, H) / \operatorname{ker} W$ and there exist positive constants $M_{1}, M_{2}$, such that $\|\Phi\| \leq M_{1}$ and $\left\|\widetilde{W}^{-1}\right\| \leq M_{2}$. 
Theorem 5.2. If hypotheses $\left(H_{1}\right) \sim\left(H_{16}\right)$ are satisfied, then the control nonlocal fractional integrodifferential system (5.1) is exactly null controllable on $I$.

Proof. Let $S_{r}=\left\{u: u \in C(I: X), u(0)-B_{4}^{-1} \int_{0}^{a} h(u(t)) d t=u_{0},\|u\| \leq r, t \in I\right\}$.

We define an operator $Q: S_{r} \rightarrow S_{r}$ by

$$
\begin{aligned}
\left(Q u_{\mu}\right)(t)= & U(t, 0 ; u) u_{0}+U(t, 0 ; u) B_{4}^{-1} \int_{0}^{a} h(u(t)) d t \\
& +\int_{0}^{t} U(t, \eta ; u) \Phi \widetilde{W}^{-1}\left[-U(a, 0 ; u) u_{0}-U(a, 0 ; u) B_{4}^{-1} \int_{0}^{a} h(u(t)) d t\right. \\
& -\int_{0}^{a} U(a, s ; u) \Psi\left(s, f\left(s, B_{2} u(s)\right),\right. \\
& \left.+\int_{0}^{t} U(t, s ; u) \Psi\left(s, f\left(s, B_{2} u(s)\right), \int_{0}^{s} g\left(s, \tau, B_{3} u(\tau)\right) d \tau\right) d s\right](\eta) d \eta
\end{aligned}
$$

Using the hypothesis $\left(\mathrm{H}_{14}\right)$, for an arbitrary function $u(\cdot)$, we define the control

$$
\begin{aligned}
\mu(t)=\widetilde{W}^{-1}[- & U(a, 0 ; u) u_{0}-U(a, 0 ; u) B_{4}^{-1} \int_{0}^{a} h(u(t)) d t \\
& \left.\quad-\int_{0}^{a} U(a, s ; u) \Psi\left(s, f\left(s, B_{2} u(s)\right), \int_{0}^{s} g\left(s, \tau, B_{3} u(\tau)\right) d \tau\right) d s\right](t) .
\end{aligned}
$$

Using this controller, we will show that the operator $Q$ has a fixed point. This fixed point is then a solution of (5.2).

Clearly, $Q_{\mu} u(a)=0$, which means that the control $\mu$ steers system (5.1) from the initial state $u_{0}$ to origin in time $a$, provided we can obtain a fixed point of the nonlinear operator $Q$.

Now, we show that $Q$ maps $S_{r}$ into itself.

We have

$$
\begin{aligned}
& \left\|\left(Q u_{\mu}\right)(t)\right\| \\
& \leq\left\|U(t, 0 ; u) u_{0}+U(t, 0 ; u) B_{4}^{-1} \int_{0}^{a} h(u(t)) d t\right\| \\
& +\int_{0}^{t}\|U(t, \eta ; u)\|\left\|\Phi \widetilde{W}^{-1}\right\|\left[\left\|U(a, 0 ; u) u_{0}+U(a, 0 ; u) B_{4}^{-1} \int_{0}^{a} h(u(t)) d t\right\|\right. \\
& +\int_{0}^{a}\|U(a, s ; u)\|\left\{\| \Psi\left(s, f\left(s, B_{2} u(s)\right), \int_{0}^{s} g\left(s, \tau, B_{3} u(\tau)\right) d \tau\right)\right. \\
& -\Psi(s, 0,0)\|+\| \Psi(s, 0,0) \|\} d s] d \eta
\end{aligned}
$$




$$
\begin{aligned}
& \quad+\int_{0}^{t}\|U(t, s ; u)\| \\
& \quad \times\left\{\left\|\Psi\left(s, f\left(s, B_{2} u(s)\right), \int_{0}^{s} g\left(s, \tau, B_{3} u(\tau)\right) d \tau\right)-\Psi(s, 0,0)\right\|+\|\Psi(s, 0,0)\|\right\} d s . \\
& \leq M_{0}\left\|u_{0}\right\|+a M_{0} C_{\alpha} k_{1} \\
& \quad+a M_{0} M_{1} M_{2}\left[M_{0}\left\|u_{0}\right\|+a M_{0} C_{\alpha} k_{1}+a M_{0}\left\{N_{1}\left(k_{5} r+k_{6}+k_{3} r+k_{4}\right)+N_{2}\right\}\right] \\
& \quad+a M_{0}\left\{N_{1}\left(k_{5} r+k_{6}+k_{3} r+k_{4}\right)+N_{2}\right\} \\
& \leq r .
\end{aligned}
$$

Thus, $Q$ maps $S_{r}$ into itself. Now, for $u, v \in S_{r}$, we have

$$
\begin{aligned}
& \left\|Q u_{\mu}(t)-Q v_{\mu}(t)\right\| \\
& \leq\left\|U(t, 0 ; u) u_{0}-U(t, 0 ; v) u_{0}\right\|+\left\|U(t, 0 ; u) B_{4}^{-1} \int_{0}^{a} h(u(t)) d t-U(t, 0 ; v) B_{4}^{-1} \int_{0}^{a} h(v(t)) d t\right\| \\
& +\int_{0}^{t}\left\{\| U(t, \eta ; u) \Phi \widetilde{W}^{-1}\left[-U(a, 0 ; u) u_{0}-U(a, 0 ; u) B_{4}^{-1} \int_{0}^{a} h(u(t)) d t\right.\right. \\
& \left.\quad-\int_{0}^{a} U(a, s ; u) \Psi\left(s, f\left(s, B_{2} u(s)\right), \int_{0}^{s} g\left(s, \tau, B_{3} u(\tau)\right) d \tau\right) d s\right] \\
& \quad-U(t, \eta ; v) \Phi \widetilde{W}^{-1}\left[-U(a, 0 ; v) u_{0}-U(a, 0 ; v) B_{4}^{-1} \int_{0}^{a} h(v(t)) d t\right. \\
& +\int_{0}^{t} \| U(t, s ; u) \Psi\left(s, f\left(s, B_{2} u(s)\right), \int_{0}^{s} g\left(s, \tau, B_{3} u(\tau)\right) d \tau\right) \\
& -U(t, s ; v) \Psi\left(s, f\left(s, B_{2} v(s)\right), \int_{0}^{s} g\left(s, \tau, B_{3} v(\tau)\right) d \tau\right) \| d s . \\
& +\left\|_{0} U(t, 0 ; v) B_{4}^{-1} \int_{0}^{a} h(u(t)) d t-U(t, 0 ; v) B_{4}^{-1} \int_{0}^{a} h(v(t)) d t\right\| \\
& +\int_{0}^{t}\left\{\| U(t, \eta ; u) \Phi \widetilde{W^{-1}}\left[-U(a, 0 ; u) u_{0}-U(s)\right),\right. \\
& \leq U(t, 0 ; u) u_{0}-U(t, 0 ; v) u_{0}\|+\| U(t, 0 ; u) B_{4}^{-1} \int_{0}^{a} h(u(t)) d t-U(t, 0 ; v) B_{4}^{-1} \int_{0}^{a} h(u(t)) d t \|
\end{aligned}
$$


Journal of Applied Mathematics

$$
\begin{gathered}
\left.\quad-\int_{0}^{a} U(a, s ; u) \Psi\left(s, f\left(s, B_{2} u(s)\right), \int_{0}^{s} g\left(s, \tau, B_{3} u(\tau)\right) d \tau\right) d s\right] \\
-U(t, \eta ; v) \Phi \widetilde{W}^{-1}\left[-U(a, 0 ; v) u_{0}-U(a, 0 ; v) B_{4}^{-1} \int_{0}^{a} h(v(t)) d t\right. \\
\quad-\int_{0}^{a} U(a, s ; v) \Psi\left(s, f\left(s, B_{2} v(s)\right),\right. \\
+\int_{0}^{t}\left\{\| U(t, s ; u) \Psi\left(s, f\left(s, B_{2} u(s)\right), \int_{0}^{s} g\left(s, \tau, B_{3} u(\tau)\right) d \tau\right)\right. \\
-U(t, s ; v) \Psi\left(s, f\left(s, B_{2} u(s)\right), \int_{0}^{s} g\left(s, \tau, B_{3} u(\tau)\right) d \tau\right) \| \\
\left.\left.\left.\left.+\| U(t, s ; v) \Psi\left(s, f\left(s, B_{2} u(s)\right), \int_{0}^{s} g\left(s, \tau, B_{3} u(\tau)\right) d \tau\right)\right) d \tau\right) d s\right] \|\right\} d \eta \\
\left.\quad-U(t, s ; v) \Psi\left(s, f\left(s, B_{2} v(s)\right), \int_{0}^{s} g\left(s, \tau, B_{3} v(\tau)\right) d \tau\right) \|\right\} d s . \\
\leq\left(K a\left\|u_{0}\right\|+a^{2} C_{\alpha} k_{1} K+a M_{0} C_{\alpha} k_{2}\right) \max _{\tau \in I}\|u(\tau)-v(\tau)\| \\
+2 a\left\{M_{0} M_{1} M_{2}\left[M_{0}\left\|u_{0}\right\|+a M_{0} C_{\alpha} k_{1}+a M_{0} N_{1}\left(k_{5}+k_{3}\right)\right]\right\} \max _{\tau \in I}\|u(\tau)-v(\tau)\| \\
+a\left\{a K\left[N_{1}\left(k_{5} r+k_{6}+k_{3} r+k_{4}\right)+N_{2}\right]+M_{0} N_{1}\left(k_{5}+k_{3}\right)\right\} \max _{\tau \in I}\|u(\tau)-v(\tau)\|
\end{gathered}
$$

Therefore, $Q$ is a contraction mapping, and hence there exists a unique fixed point $u \in X$, such that $Q u(t)=u(t)$. Any fixed point of $Q$ is a mild solution of (5.1) on $I$ which satisfies $u(a)=0$. Thus, system (5.1) is exactly null controllable on $I$.

\section{Examples}

To illustrate the abstract results, we give the following examples.

Example 6.1. Consider the nonlinear integropartial differential equation of fractional order

$$
\frac{\partial^{\alpha} u(x, t)}{\partial t^{\alpha}}+\sum_{|q| \leq 2 m} a_{q}\left(x, t ; b_{q} u(x, t)\right) D_{x}^{q} u(x, t)=F\left(x, t, w_{1}\right)+\int_{0}^{t} G\left(x, t, s, w_{2}(s)\right) d s,
$$


with nonlocal condition

$$
u(x, 0)+\sum_{k=1}^{p} c_{k} u\left(x, t_{k}\right)=g(x)
$$

where $0<\alpha \leq 1,0 \leq t_{1}<\cdots<t_{p} \leq a, x \in R^{n}, D_{x}^{q}=D_{x_{1}}^{q_{1}} \cdots D_{x_{n}}^{q_{n}}, D_{x_{i}}=\partial / \partial x_{i}, q=\left(q_{1}, \ldots, q_{n}\right)$ is an $n$-dimensional multi-index, $|q|=q_{1}+\cdots+q_{n}$, and $w_{i}, i=1,2$, is given by

$$
w_{i}(x, t)=\sum_{|q| \leq 2 m-1} b_{q_{i}}(x, t) D_{x}^{q} u(x, t)+\int_{\Omega} \sum_{|q| \leq 2 m-1} c_{q_{i}}(x, \mathfrak{t}) D_{y}^{q} u(y, t) d y
$$

Let $L_{2}\left(R^{n}\right)$ be the set of all square integrable functions on $R^{n}$. We denote by $C^{m}\left(R^{n}\right)$ the set of all continuous real-valued functions defined on $R^{n}$ which have continuous partial derivatives of order less than or equal to $m$. By $C_{0}^{m}\left(R^{n}\right)$, we denote the set of all functions $f \in C^{m}\left(R^{n}\right)$ with compact supports. Let $H^{m}\left(R^{n}\right)$ be the completion of $C_{0}^{m}\left(R^{n}\right)$ with respect to the norm

$$
\|f\|_{m}^{2}=\sum_{|q| \leq m} \int_{R^{n}}\left|D_{x}^{q} f(x)\right|^{2} d x .
$$

It is supposed that the following hold.

(i) The operator $A=-\sum_{|q| \leq 2 m} a_{q}\left(x, t ; b_{q} u(x, t)\right) D_{x}^{q}$ is uniformly elliptic on $R^{n}$. In other words, all the coefficients $a_{q},|q|=2 m$, are continuous and bounded on $R^{n}$, and there is a positive number $c$ such that

$$
(-1)^{m+1} \sum_{|q|=2 m} e_{q}\left(x, t ; b_{q}\right) \xi^{q} \geq c|\xi|^{2 m}
$$

for all $x \in R^{n}$ and all $\xi \neq 0, \xi \in R^{n}, \xi^{q}=\xi_{1}^{q_{1}} \cdots \xi_{n}^{q_{n}}$, and $|\xi|^{2}=\xi_{1}^{2}+\cdots+\xi_{n}^{2}$.

(ii) All the coefficients $a_{q},|q|=2 m$, satisfy a uniform Hölder condition on $R^{n}$. Under these conditions, the operator $A$ with domain of definition $D(A)=H^{2 m}\left(R^{n}\right)$ generates an evolution operator defined on $L_{2}\left(R^{n}\right)$, and it is well known that $H^{2 m}\left(R^{n}\right)$ is dense in $X=$ $L_{2}\left(R^{n}\right)$ and the initial function $g(x)$ is an element in Hilbert space $H^{2 m}\left(R^{n}\right)$, see [26, page 438]. Applying Theorem 4.1, this achieves the proof of the existence of mild solutions of the problem (6.1), (6.2). In addition,

(iii) If the coefficients $b_{q}, c_{q},|q| \leq 2 m-1$ satisfy a uniform Hölder condition on $R^{n}$ and the operators $F$ and $G$ satisfy.

There are numbers $L_{1}, L_{2} \geq 0$ and $\lambda_{1}, \lambda_{2} \in(0,1)$ such that

$$
\begin{gathered}
\sum_{|q| \leq 2 m-1} \int_{R^{n}}\left|F\left(x, t, D_{x}^{q} w_{1}\right)-F\left(x, s, D_{x}^{q} w_{1}^{*}\right)\right|^{2} d x \leq L_{1}\left(|t-s|^{\lambda_{1}}+\left|w_{1}-w_{1}^{*}\right|^{2} d x\right), \\
\sum_{|q| \leq 2 m-1} \int_{R^{n}}\left|G\left(x, t, \eta, D_{x}^{q} w_{2}\right)-G\left(x, s, \eta, D_{x}^{q} w_{2}\right)\right|^{2} d x \leq L_{2}|t-s|^{\lambda_{2}},
\end{gathered}
$$


for all $t, s \in I,(t, \eta),(s, \eta) \in \Delta$, and all $x \in R^{n}$. Applying Theorem 4.2, we deduce that (6.1), (6.2) has a unique strong solution.

The second example is concerned with the controllability result.

Example 6.2. Consider the fractional nonlocal evolution integropartial differential control system of the form

$$
\begin{gathered}
\frac{\partial^{\alpha} u(x, t)}{\partial t^{\alpha}}+a(x, t,(b u)(x, t)) \frac{\partial^{2} u(x, t)}{\partial x^{2}}=\zeta(x, t)+\Upsilon\left(t, \mu_{1}(t, u(x, t)), \int_{0}^{t} \mu_{2}(t, s, u(x, t)) d s\right), \\
u(x, 0)+\sum_{k=1}^{p} c_{k} u\left(x, t_{k}\right)=g(x), \quad x \in[0, \pi], \\
u(0, t)=u(\pi, t)=0, \quad t \in I,
\end{gathered}
$$

where $0<\alpha \leq 1,0 \leq t_{1}<\cdots<t_{p} \leq a$, and the functions $a(x, t, \cdot), b(x, t)$ are continuous.

Let us take

$$
X=L^{2}[0, \pi], \quad S_{r}=\left\{y \in L^{2}[0, \pi]:\|y\| \leq r\right\}
$$

Put $(\Phi \mu)(x)(t)=\zeta(x, t), x \in[0, \pi]$ where $\mu(t)=\zeta(\cdot, t)$ and $\zeta:[0, \pi] \times I \rightarrow[0, \pi]$ is continuous.

We define $A(t, \cdot): X \rightarrow X$ by $(A(t, \cdot) w)(x)=a(x, t, \cdot) w^{\prime \prime}$ with domain $D(A)=\{w \in X:$ $w, w^{\prime}$ are absolutely continuous, $\left.w^{\prime \prime} \in X, w(0)=w(\pi)=0\right\}$. Assume that $-A(t, \cdot)$ generates an evolution system $U(t, s, \cdot)$ such that for every positive numbers $n_{1}$ and $n_{2},\|U(t, s, \cdot)\| \leq n_{1}$ and $\|U(t, s, \cdot) A(s, \cdot)\| \leq n_{2}$. $x \in[0, \pi]$.

Also, define $B_{1}(t): D\left(B_{1}\right)=S_{1} \rightarrow X$ by $\left(B_{1}(t) z\right)(x)=b(x, t) z$, for all $z \in X$ and

Assume that the linear operator $W$ that is given by

$$
W \mu(x)=\int_{0}^{a} U(a, s ; u) \zeta(x, s) d s, \quad x \in[0, \pi]
$$

has a bounded invertible operator $\widetilde{W}^{-1}$ in $L^{2}(I, H) / \operatorname{ker} W$.

Let us assume that the nonlinear functions $\Upsilon, \mu_{1}$, and $\mu_{2}$ satisfy the following Lipschitz conditions

$$
\begin{gathered}
\left\|\Upsilon\left(t, z_{1}, y_{1}\right)-\Upsilon\left(t, z_{2}, y_{2}\right)\right\| \leq c_{1}\left[\left\|z_{1}-z_{2}\right\|+\left\|y_{1}-y_{2}\right\|\right], \\
\left\|\mu_{1}\left(t, x_{1}\right)-\mu_{1}\left(t, x_{2}\right)\right\| \leq c_{2}\left\|x_{1}-x_{2}\right\|, \\
\int_{0}^{t}\left\|\mu_{2}\left(t, s, v_{1}\right)-\mu_{2}\left(t, s, v_{2}\right)\right\| d s \leq c_{3}\left\|v_{1}-v_{2}\right\|,
\end{gathered}
$$

where $c_{j}>0, j=1,2,3, x_{i}, y_{i}, z_{i}, v_{i} \in X, i=1,2$, and $s, t \in I$. 
All the conditions stated in Theorem 5.2 are satisfied. Hence, system (6.7) is exactly null controllable on $I$.

\section{References}

[1] D. Baleanu, R. P. Agarwal, O. G. Mustafa, and M. C. Sulschi, "A symptotic integration of some nonlinear differential equations with fractional time derivative," Journal of Physics A, vol. 44, pp. 1-9, 2011.

[2] D. Baleanu and A. K. Golmankhaneh, "On electromagnetic field in fractional space," Nonlinear Analysis. Real World Applications, vol. 11, no. 1, pp. 288-292, 2010.

[3] A. A. Kilbas, H. M. Srivastava, and J. J. Trujillo, Theory and Applications of Fractional Differential Equations, vol. 204 of North-Holland Mathematics Studies, Elsevier Science B.V., Amsterdam, The Netherlands, 2006.

[4] S. G. Samko, A. A. Kilbas, and O. I. Marichev, Fractional Integrals and Derivatives: Theory and Applications, Gordon and Breach Science, Yverdon, Switzerland, 1993.

[5] I. Podlubny, Fractional Differential Equations, vol. 198 of Mathematics in Science and Engineering, Technical University of Kosice, Kosice, Slovak Republic, 1999.

[6] B. Zhu, "The space fractional diffusion equation with Feller's operator," Applied Mathematics and Computation, vol. 217, no. 24, pp. 9971-9977, 2011.

[7] E. Hernández, D. O’Regan, and K. Balachandran, “On recent developments in the theory of abstract differential equations with fractional derivatives," Nonlinear Analysis, vol. 73, no. 10, pp. 3462-3471, 2010.

[8] D. Băleanu and O. G. Mustafa, "On the global existence of solutions to a class of fractional differential equations," Computers \& Mathematics with Applications, vol. 59, no. 5, pp. 1835-1841, 2010.

[9] B. Ahmad and R. P. Agarwal, "On nonlocal fractional boundary value problems," Dynamics of Continuous, Discrete E Impulsive Systems A, vol. 18, no. 4, pp. 535-544, 2011.

[10] D. Baleanu, K. Diethelm, E. Scalas, and J. J. Trujillo, Fractional Calculus Models and Numerical Methods, Series on Complexity, Nonlinearity and Chaos, World Scientific, Boston, Mass, USA, 2012.

[11] B. Ahmad and J. J. Nieto, "Riemann-Liouville fractional integro-differential equations with fractional nonlocal integral boundary conditions," Boundary Value Problems, vol. 2011, article 36, 9 pages, 2011.

[12] B. Ahmad and S. K. Ntouyas, "A four-point nonlocal integral boundary value problem for fractional differential equations of arbitrary order," Electronic Journal of Qualitative Theory of Differential Equations, no. 22, 15 pages, 2011.

[13] X. B. Shu, Y. Z. Lai, and Y. Chen, "The existence of mild solutions for impulsive fractional partial differential equations," Nonlinear Analysis, vol. 74, pp. 2003-2011, 2011.

[14] R. Almeida and D. F. M. Torres, "Necessary and sufficient conditions for the fractional calculus of variations with Caputo derivatives," Communications in Nonlinear Science and Numerical Simulation, vol. 16, no. 3, pp. 1490-1500, 2011.

[15] X. Fu, "Controllability of neutral functional differential systems in abstract space," Applied Mathematics and Computation, vol. 141, no. 2-3, pp. 281-296, 2003.

[16] X. Fu, "Controllability of abstract neutral functional differential systems with unbounded delay," Applied Mathematics and Computation, vol. 151, no. 2, pp. 299-314, 2004.

[17] A. Debbouche and D. Baleanu, "Controllability of fractional evolution nonlocal impulsive quasilinear delay integro-differential systems," Computers $\mathcal{E}$ Mathematics with Applications, vol. 62, no. 3, pp. 14421450, 2011.

[18] L. Byszewski, "Theorems about the existence and uniqueness of solutions of a semilinear evolution nonlocal Cauchy problem," Journal of Mathematical Analysis and Applications, vol. 162, no. 2, pp. 494$505,1991$.

[19] L. Byszewski, "Application of properties of the right-hand sides of evolution equations to an investigation of nonlocal evolution problems," Nonlinear Analysis. Theory, Methods E Applications A, vol. 33, no. 5, pp. 413-426, 1998.

[20] A. Debbouche, "Fractional nonlocal impulsive quasilinear multi-delay integro-differential systems," Advances in Difference Equations, vol. 5, pp. 1-10, 2011.

[21] A. Debbouche, "Fractional evolution integro-differential systems with nonlocal conditions," Advances in Dynamical Systems and Applications, vol. 5, no. 1, pp. 49-60, 2010.

[22] G. M. N'Guérékata, "A Cauchy problem for some fractional abstract differential equation with non local conditions," Nonlinear Analysis. Theory, Methods \& Applications A, vol. 70, no. 5, pp. 1873-1876, 2009. 
[23] Z. Yan, "Controllability of semilinear integrodifferential systems with nonlocal conditions," International Journal of Computational and Applied Mathematics, vol. 2, no. 3, pp. 221-236, 2007.

[24] K. Deng, "Exponential decay of solutions of semilinear parabolic equations with nonlocal initial conditions," Journal of Mathematical Analysis and Applications, vol. 179, no. 2, pp. 630-637, 1993.

[25] A. Pazy, Semigroups of Linear Operators and Applications to Partial Differential Equations, Springer, 1983.

[26] M. M. El-Borai, "Some probability densities and fundamental solutions of fractional evolution equations," Chaos, Solitons and Fractals, vol. 14, no. 3, pp. 433-440, 2002.

[27] A. Debbouche and M. M. El-Borai, "Weak almost periodic and optimal mild solutions of fractional evolution equations," Electronic Journal of Differential Equations, vol. 46, pp. 1-8, 2009.

[28] S. D. Zaidman, Abstract Differential Equations, Pitman, San Francisco, Calif, USA, 1979.

[29] R. Sakthivel, Q. H. Choi, and S. M. Anthoni, "Controllability result for nonlinear evolution integrodifferential systems," Applied Mathematics Letters, vol. 17, no. 9, pp. 1015-1023, 2004.

[30] R. Sakthivel, S. M. Anthoni, and J. H. Kim, "Existence and controllability result for semilinear evolution integrodifferential systems," Mathematical and Computer Modelling, vol. 41, no. 8-9, pp. 10051011, 2005. 


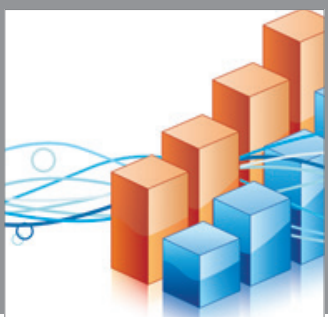

Advances in

Operations Research

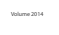

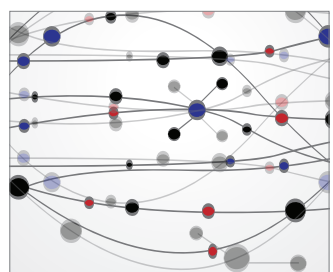

\section{The Scientific} World Journal
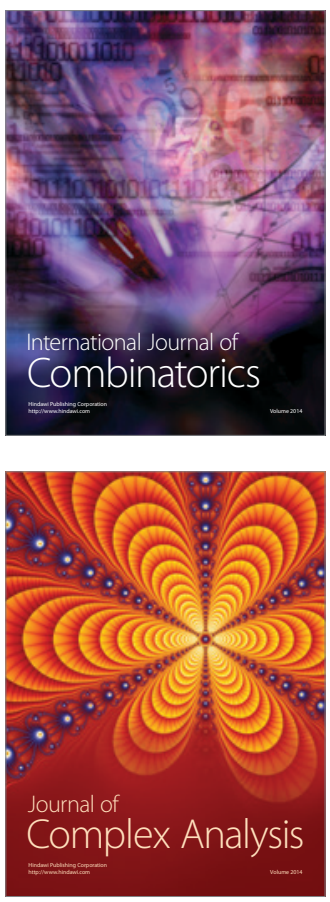

International Journal of

Mathematics and

Mathematical

Sciences
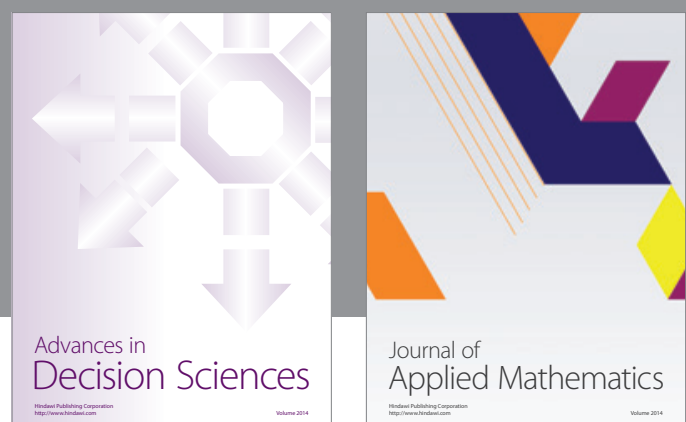

Journal of

Applied Mathematics
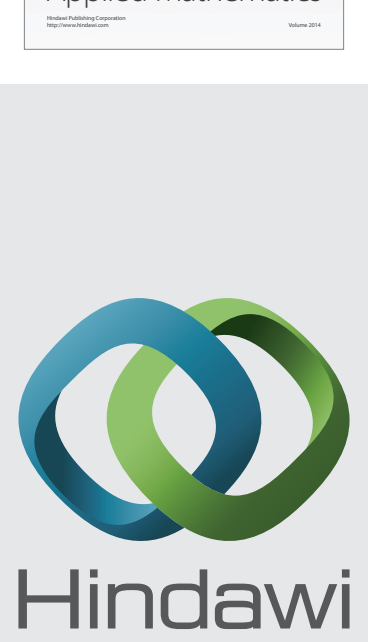

Submit your manuscripts at http://www.hindawi.com
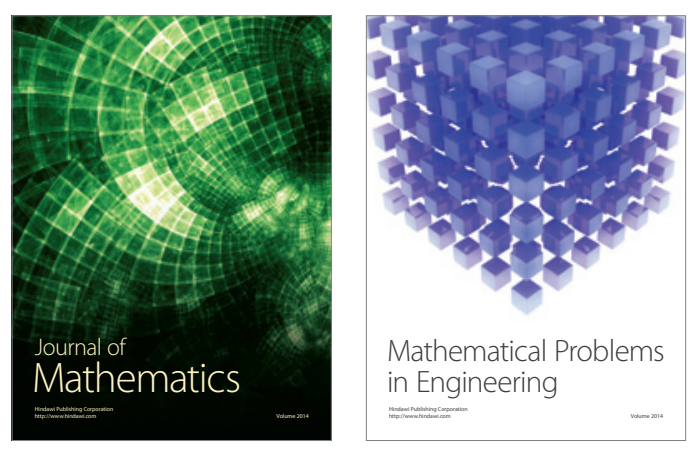

Mathematical Problems in Engineering
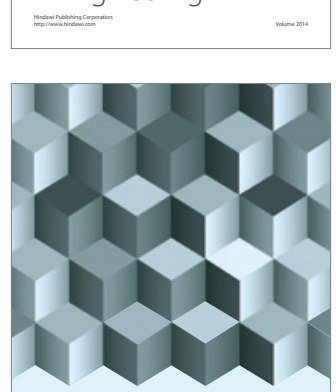

Journal of

Function Spaces
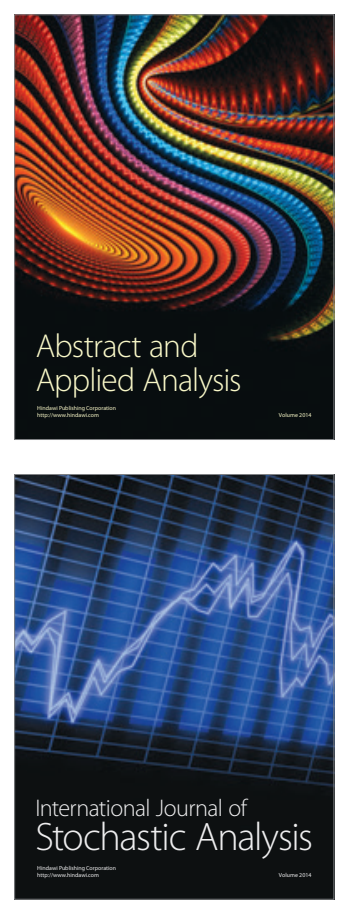

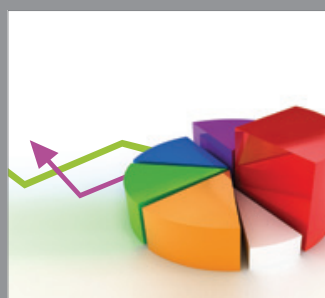

ournal of

Probability and Statistics

Promensencen
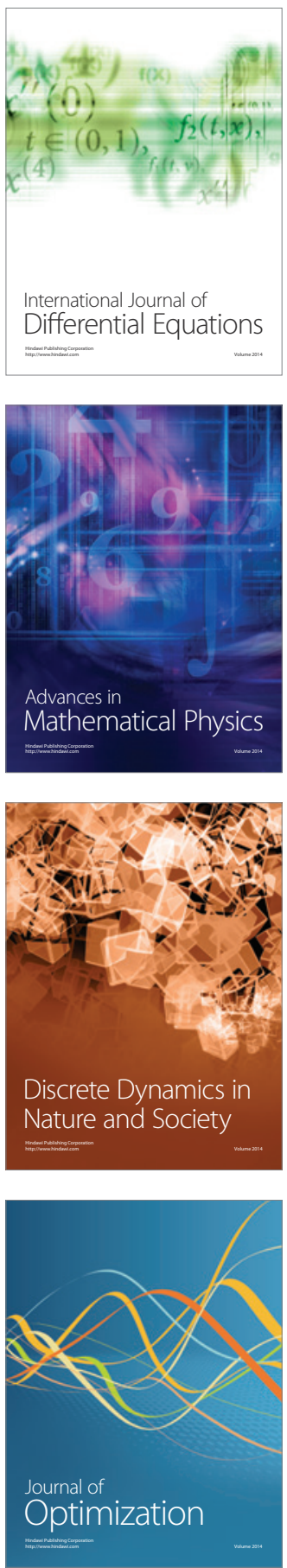